\title{
EDUCACIÓN EMOCIONAL EN LOS PROFESORES DE EDUCACIÓN INFANTIL: ASPECTO CLAVE EN EL DESEMPEÑO DOCENTE
}

\author{
María del Camino Escolar Llamazares \\ Tamara de la Torre Cruz \\ Jonathan Huelmo García \\ Carmen Palmero Cámara \\ Universidad de Burgos
}

\begin{abstract}
RESUMEN: Partiendo de la importancia que la educación emocional tiene en los alumnos de Educación Infantil resulta obligado que estas competencias también estén presentes en la formación de los docentes. El objetivo de este trabajo es analizar la oferta formativa sobre educación emocional impartida desde los CFIEs de Burgos y Miranda de Ebro. Se realizó un estudio descriptivo y transversal. Se llevaron a cabo análisis de frecuencias con el programa estadístico SPPS v. 22. Se obtiene que el $69,9 \%$ de los cursos analizados están relacionados con la educación emocional o inteligencia emocional, que este tipo de cursos se ha triplicado en los últimos años, siendo los centros públicos lo que en su mayoría los realizan. Los destinatarios principales son el profesorado de las etapas de infantil y primaria. La educación emocional en el desempeño docente del profesorado de Educación Infantil es un aspecto clave para que éstos puedan implementar metodologías activas que posibiliten el desarrollo integral del alumnado. Por lo tanto, pese a que la oferta de formación permanente vinculada a las habilidades emocionales se está incrementando en los últimos años, es necesario que los responsables de los CFIEs planifiquen un plan de actuación que se desarrolle en todas las zonas de la provincia de Burgos.
\end{abstract}

PALABRAS CLAVE: Educación emocional, desempeño docente, Educación Infantil, maestros.

\section{EMOTIONAL EDUCATION IN EARLY CHILDHOOD EDUCATION TEACHERS: A KEY ASPECT IN TEACHING PERFORMANCE}

ABSTRACT: Based on the importance of emotional education in Early Childhood students it is equally important that these skills are also present in the training of teachers. The objective is to analyze the training offered by 
the CFIEs of Burgos and Miranda de Ebro to Early Childhood teachers about emotional education. This is a descriptive cross-sectional study. In relation to the results, the analysis focuses on the study of frequencies using the SPPS statistical program v. 22. Results: We obtain that $69.9 \%$ of the courses taken include in the title a term related to emotional education or emotional intelligence, that such courses has tripled in recent years, public schools being mostly what carried them. The main beneficiaries are the teachers of the Early Childhood and primary education. In conclusion, the emotional education in the performance of teachers of Early Childhood it is a key aspect so that they can implement active methodologies that facilitate the overall development of students, so despite the continuing education program related to these aspects is increasing in recent years, it would require that responsible for CFIEs establish an action plan to develop in all areas of the province of Burgos.

KEYWORDS: Emotional education, teacher performance, Early Childhood Education, teachers.

Recibido: 21/03/2016

Aceptado: 15/07/2016

Correspondencia: María del Camino Escolar Llamazares, Facultad de Ciencias de la Salud, Universidad de Burgos, Po Comendadores, s/n (Hospital Militar), 09001 Burgos. Email: cescolar@ubu.es.

\section{INTRODUCCIÓN}

El objetivo fundamental de la educación y en concreto de la Educación Infantil, es conseguir un desarrollo integral y equilibrado de la personalidad de los alumnos (Bisquerra, 2000; Gutiérrez, Ibañez, y Aguilar, 2013; Palomero Pescador, 2005), en este desarrollo las emociones juegan un papel fundamental.

Como sabemos, las emociones son responsables de nuestras decisiones, tanto acertadas como desacertadas y son indispensables para la racionalidad (Damasio, 2001; Sáenz-López Buñuel y Heras Pérez, 2013). Esto hace que debamos ocuparnos del mundo emocional de nuestros educandos, a través de lo que Bisquerra (2005) denomina la educación emocional.

La educación emocional es entendida como un proceso educativo, continuo y permanente que potencia el desarrollo de las competencias emocionales (Bisquerra, 2005), entre ellas la Inteligencia Emocional (IE) (Goleman, 1995; Mayer, Salovey, y Caruso, 2010). Dada su importancia, autores como Mayer y Salovey (1997) ya sugirieron la inclusión de la enseñanza emocional dentro del currículo escolar (García-Martínez, Fernández Ozcorta, Rodríguez Peláez, y Tornero Quiñones, 2013) convirtiéndosela escuela en uno de los medios más importantes a través del cual el niño aprende y conforma su personalidad. Por lo tanto, es en los centros educativos donde se debe enseñar al alumnado a ser emocionalmente 
más inteligente, dotándole de estrategias y habilidades básicas que les protejan de los factores de riesgo, o al menos, que palien sus efectos negativos (De Andrés Viloria, 2005).

La gestión de las emociones en el contexto escolar ha tenido una notable incidencia en las últimas décadas, dando lugar a numerosos estudios, que verifican la importancia y la idoneidad de la aplicación de programas que fomenten la educación socioemocional en el alumnado (Durlaky Weissberg, 2005; Fernández-Berrocal, Extremera y Palomera, 2008; García Martínez et al., 2013; Qualter, Gardner, Pope, y Kelly, 2012; Mayer y Salovey, 1997; Palomero Pescador, 2005).

En este contexto, la mayoría de los docentes consideran primordial el desarrollo de habilidades emocionales para lograr el desarrollo integral de sus alumnos (Barberá y Fuertes, 2012; Cassullo y García, 2015). Sin embargo, las habilidades emocionales, afectivas y sociales deben ser enseñadas por un profesorado que domine estas capacidades (Cabello, Ruiz-Aranda, y Fernández-Berrocal, 2010; Extremera y Fernández-Berrocal, 2004a; Palomera, Fernández-Berrocal, y Brackett, 2008; Poulou, 2005). Es decir, que para poder aplicar estos programas de forma efectiva en el aula, es necesario que los docentes tengan un desarrollo elevado de competencias socioemocionales (Extremera y Fernández-Berrocal, 2004a,b,c), que por otra parte les permitirá relacionarse de forma positiva y adecuada con la comunidad educativa, aumentando la eficacia y eficiencia de la educación (Bisquerra, 2007; Ramírez y De la Herrán, 2012). Por lo tanto, se requiere para los profesores una formación teórico-práctica previa (Bisquerra, 2005; Conde y Almagro, 2013).

Esta formación, tal y como señalan numerosos autores tendría que ser continua, es decir, es fundamental que la educación emocional esté presente en los procesos de formación inicial y permanente de los docentes de Educación Infantil (Bisquerra, 2000, 2005; Palomero Pescador, 2005; Punset, 2010; Sáenz-López Buñuel y Heras Pérez, 2013). Pues los maestros, social y emocionalmente competentes, desarroIlan su docencia a través del apoyo a sus alumnos, potenciando sus habilidades y fortalezas, la cooperación y la comunicación; además de aplicar estrategias que desarrollan la motivación intrínseca (Jennings y Greenberg, 2009; García Martínez et al., 2013).

En lo referente a la formación inicial algunos autores consideran acertada la inclusión de las competencias emocionales en el currículo universitario, si bien, en la práctica ocupan, en general, un segundo plano dentro del conjunto de las competencias docentes (Bisquerra, 2003, 2007; Extremera y Fernández-Berrocal, 2005; Gutiérrez et al., 2013; López-Goñi y Goñi, 2012; Molero, Ortega, y Moreno, 2010; Palomera et al., 2008; Pegalajar Palomino y López Hernáez, 2015).

En lo referente a la formación permanente, teniendo en cuenta el Decreto 51/2014 de 9 de octubre, ésta ha de ser entendida como una instrucción encaminada al desarollo personal y profesional del profesorado, para que pueda responder a las funciones que la sociedad le encomienda.

En la mayoría de los casos, el desarrollo de competencias emocionales de forma intencional y sistemática suele estar ausente en los programas de desempeño docente de los maestros (Bisquerra, 2005) de Educación Infantil. De hecho, en España existen pocos programas dirigidos a fomentar la práctica emocional del profesora- 
do (Fernández, Palomero, y Teruel, 2009), formación que además de insuficiente suele ser demasiado teórica y poco práctica (Barrantes, Casas, y Luengo, 2011).

Estas carencias formativas en competencias emocionales, son detectadas por los maestros, cuyas demandas formativas son recogidas desde los Centros de Formación del Profesorado e Innovación Educativa (CFIEs), órganos de referencia para el profesorado en materia de formación permanente (Decreto 51/2014 de 9 de octubre).

El objetivo de este trabajo es analizarla oferta formativa continuada sobre educación emocional que se imparte desde los CFIEs de Burgos y Miranda de Ebro al profesorado de Educación Infantil, teniendo en cuenta que la educación emocional es clave para el desarrollo integral del alumnado en esta etapa y que el maestro es el principal transmisor de estas competencias en el aula.

\section{Método}

La investigación que se presenta es un estudio descriptivo y transversal mediante un código arbitrario de observación construido previamente, tal y como sugieren Montero y León (2007), con el objetivo de analizar sistemáticamente los datos sobre formación en educación emocional facilitados por los dos CFIEs de la provincia de Burgos.

\section{Resultados}

Para dar satisfacción al objetivo planteado se analizan los datos ofrecidos por los CFIEs sobre las actividades de formación continua impartidos desde el curso 20102011 hasta la actualidad en Burgos y provincia, que tengan presente en su título y/o en los descriptores las palabras de inteligencia emocional o educación emocional. El proceso de análisis realizado se centra en el estudio de frecuencias utilizando para ello el programa estadístico SPPS v. 22.

Los datos ofrecidos por el CFIE se clasifican en los parámetros que se exponen en la tabla 1.

Del volumen de información analizada nos hemos centrado en 73 acciones de formación continua realizadas por los CFIEs que cumplen con la presencia en el título y/o en los descriptores del concepto de educación emocional o IE, siendo la distribución de la muestra en función de esta variable la correspondiente a la figura 1. En ella podemos observar como en el 69,9\% de la muestra analizada se incluye dentro del título alguno de estos términos, lo que sitúa el concepto como necesidad formativa, ya que no es incluido como tema en otros cursos, sino que adquiere entidad y relevancia propia al ser núcleo central entorno al que se articula una actividad formativa del profesorado.

Teniendo en consideración la oferta de formación sobre educación emocional por curso académico, figura 2, podemos destacar dos aspectos: por un lado el descenso del curso 2010-2011 al 2011-2012 de 11 a 7 cursos y, por otro lado, la evolución continua en la oferta desde el curso académico 2011-2012, llegando a triplicarse en la actualidad, 2015-2016, así se ha pasado de 7 a 21 cursos. Estos datos muestran el interés de los docentes por el tema. 
Tabla 1. Variables de estudio de las actividades de formación continua ofrecidas por el CFIE

\begin{tabular}{|c|c|}
\hline PARÁMETRO & DESCRIPCIÓN \\
\hline Inteligencia Emocional & $\begin{array}{l}\text { Presencia de los conceptos Inteligencia Emocional en el } \\
\text { título y/o en los descriptores. }\end{array}$ \\
\hline Cursos realizados & $\begin{array}{l}\text { Número de cursos realizados por cursos desde el año } \\
\text { académico 2010-2011. }\end{array}$ \\
\hline Centro de realización & $\begin{array}{l}\text { Se recoge si el curso ha sido realizado en un centro de } \\
\text { titularidad pública, concertada o en los CFIE de la provincia } \\
\text { de Burgos. } \\
\text { En este último caso, el profesorado que participa en los } \\
\text { mismos puede proceder, indistintamente, de cualquiera } \\
\text { de las otras dos modalidades de centros presentados con } \\
\text { anterioridad. }\end{array}$ \\
\hline Etapa educativa & $\begin{array}{l}\text { Los profesores destinatarios de la formación han sigo } \\
\text { agrupados teniendo en cuenta las siguientes categorías: } \\
\text { Maestros de Educación Infantil y/o Primaria, profesorado de } \\
\text { secundaria y/o formación profesional y otros (docentes de } \\
\text { Escuela Oficial de Idioma, de Enseñanzas Artísticas...). }\end{array}$ \\
\hline Lugares de realización & $\begin{array}{l}\text { Los cursos realizados han sido impartidos en diferentes } \\
\text { centros educativos que pertenecen al ámbito de actuación } \\
\text { del CFIE de Burgos o de Miranda de Ebro. }\end{array}$ \\
\hline Eje de formación & $\begin{array}{l}\text { La oferta de formación continua puede ser realizada como } \\
\text { parte de un itinerario formativo de un Plan de formación } \\
\text { de centro, Experiencias de Calidad de centro o Planes de } \\
\text { actuación de los CFIE. }\end{array}$ \\
\hline \multirow[t]{5}{*}{ Modalidad de formación } & $\begin{array}{l}\text { En función del grado de participación y/o dotación } \\
\text { económica asociada a la actividad se han distinguido las } \\
\text { siguientes modalidades: }\end{array}$ \\
\hline & $\begin{array}{l}\text { - Cursos: La carga de la formación recae en un experto y } \\
\text { se adjudica una dotación económica. }\end{array}$ \\
\hline & $\begin{array}{l}\text { - Seminarios: Pueden contar con colaboración de } \\
\text { un experto, pero no puede exceder más del } 20 \% \text { de } \\
\text { la duración total de la actividad, y se adjudica una } \\
\text { dotación económica. }\end{array}$ \\
\hline & $\begin{array}{l}\text { - Grupos de trabajo: Regulan prácticas de intercambio } \\
\text { de experiencias y conocimientos entre profesorado } \\
\text { de la propia institución, sin colaboración de expertos } \\
\text { externo, y se adjudica una dotación económica. }\end{array}$ \\
\hline & $\begin{array}{l}\text { - Experiencias de Calidad: Regulan prácticas de } \\
\text { intercambio de experiencias entre profesorado de la } \\
\text { propia institución para mejorar un aspecto deficitario, } \\
\text { pudiendo contar con la colaboración de expertos } \\
\text { externo, y no se adjudica una dotación económica. }\end{array}$ \\
\hline
\end{tabular}




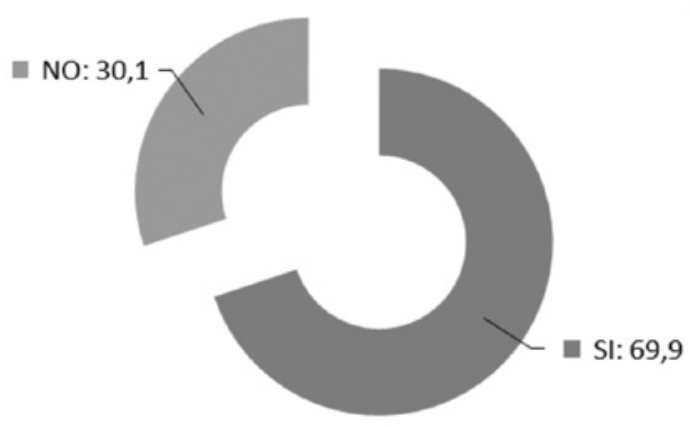

Figura 1. Presencia de los conceptos de educación emocional o Inteligencia Emocional en el título y/o en los descriptores

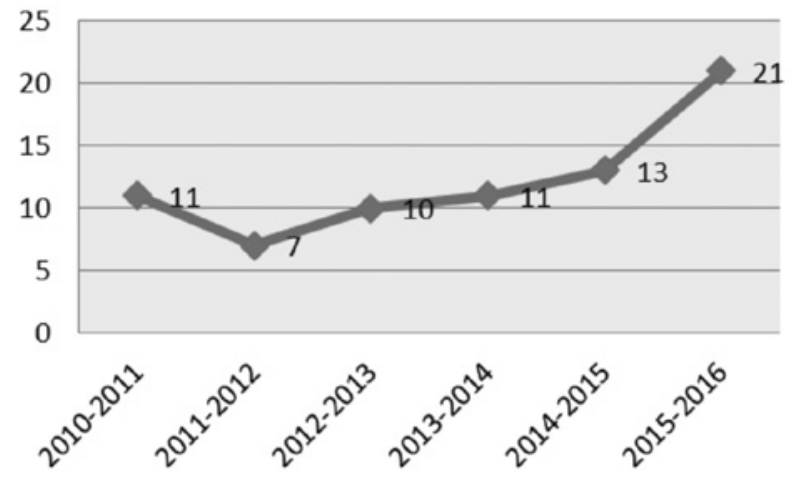

Figura 2. Cursos sobre educación emocional y/o Inteligencia Emocional por curso académico

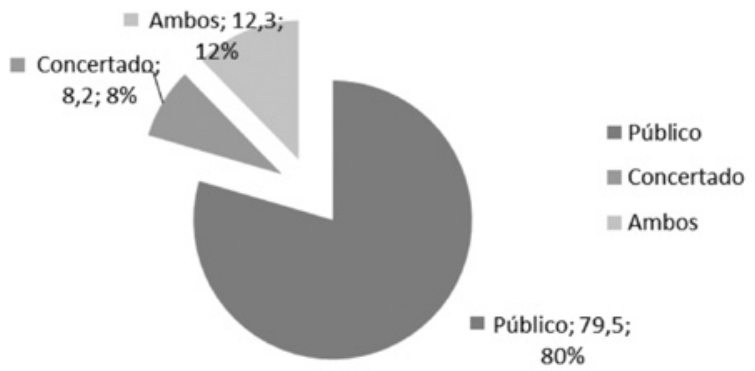

Figura 3. Titularidad de los centros donde se han realizado la formación sobre educacional emocional 
Los centros en los que se ha realizado este tipo de formación, figura 3, son en su mayoría de titularidad pública, $80 \%$, pudiendo verse incrementado este porcentaje si consideramos que en los tipificados como ambos, $12 \%$, acuden profesorado tanto de los centro públicos como concertados.

En la figura 4 se puede observar la etapa educativa de los profesores destinatarios de la formación. Así el 70\% de los cursos sobre IE tienen como destinatario los profesores de las etapas de infantil y primaria, el 18\% de la etapa de secundaria, el $11 \%$ de infantil, primaria y secundaria, y el $1,4 \%$ se refiere a docentes de otras etapas educativas.

La singular orografía de la provincia de Burgos y su distribución poblacional hace que la oferta de formación continua no se pueda circunscribir al CFIE enclavado en la capital de la provincia de Burgos, por lo que su distribución territorial ha de compensarse con la oferta de formación en el ámbito rural. En el tema que nos ocupa, la realización de los cursos sobre educación emocional o IE, la distribución de la oferta entre el ámbito urbano y rural es equitativa, se encuentra sobre el 50\%. En la figura 5 tenemos el detalle del número de cursos realizados en cada una de los centros según su localización geográfica.

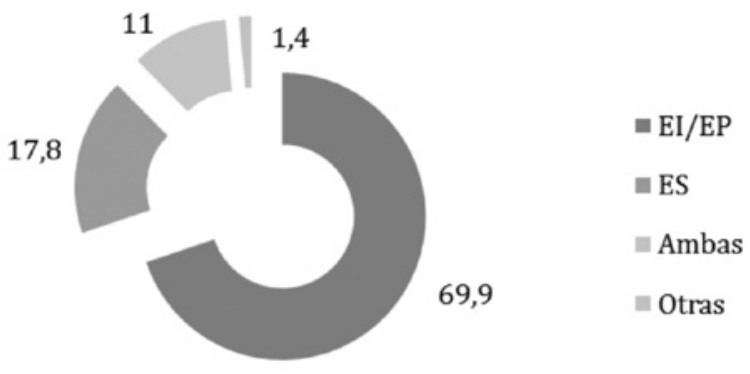

Figura 4. Etapa educativa de los profesores destinatarios de la formación

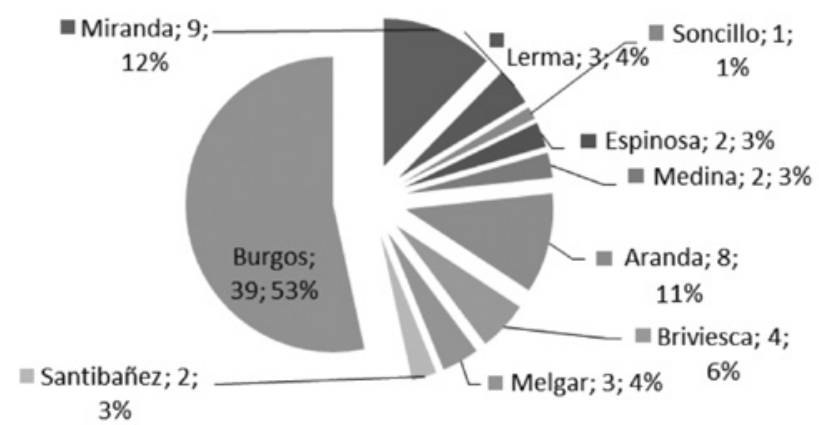

Figura 5. Número de cursos realizados por localidad 
De forma mayoritaria los cursos sobre educación emocional se han realizado como parte del itinerario formativo del Plan de Formación del centro (PF), Ilegando al $70 \%$ de la oferta analizada, tal y como se puede observar en la figura 6 . El resto de cursos se enmarcan en las experiencias de calidad de centro $(17,8 \%)$ y en los planes de actuación del CFIE (12,3\%).

La principal modalidad de formación diseñada para impartir los cursos es la de seminario, con un $43,8 \%$ del total, seguida del plan de mejora del centro con un 21 , $9 \%$, siendo éste propuesto tras la necesidad detectada por parte de los docentes del centro, de ahí su importancia de cara a considerar el conocimiento propio de los implicados sobre sus necesidades formativas. En la figura 7 se puede ver la distribución según el porcentaje.

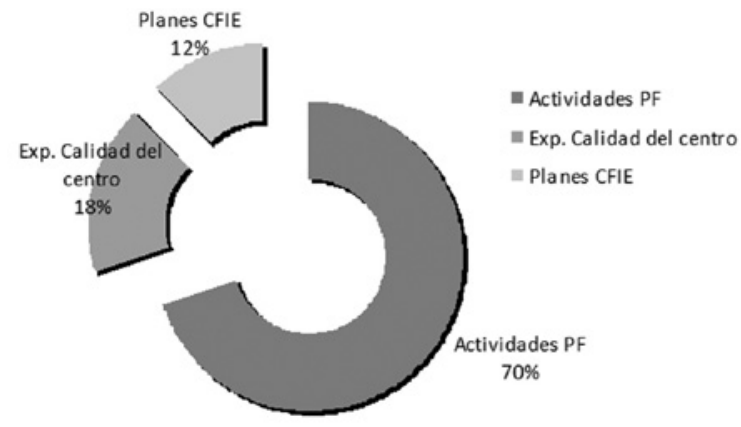

Figura 6. Eje de formación en la que se sitúa la oferta de cursos de IE

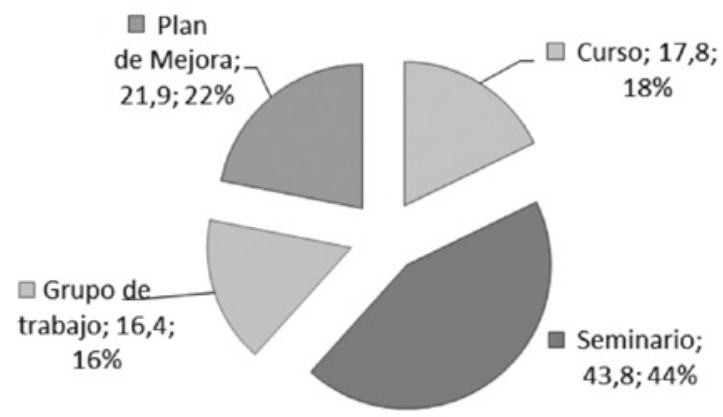

Figura 7. Modalidad de formación de los cursos de educación emocional o Inteligencia Emocional

\section{Discusión}

En función de los resultados alcanzados tras el análisis de datos podemos decir que la IE es considerada como una habilidad clave en el desarrollo de la práctica docente 
por parte del profesorado y, por ende, una competencia relevante a la hora de valorar su desempeño docente. En este sentido, los profesionales de la educación han triplicado, en los últimos cinco años la demanda de actuaciones encaminadas al conocimiento y aplicación de este concepto en su realidad educativa. Esta demanda de formación a los CEFIEs se ha materializado en forma de cursos cuya duración mínima estimada es de 10 horas, llegando a alcanzar hasta 40 horas en algunos de los analizados.

Así mismo, es destacable que la oferta de formación continua en este ámbito es mayoritariamente para los docentes de las etapas de infantil y primaria, lo que muestra la importancia de recibir formación en aspectos clave de la IE en estas etapas, ya que son fundamentales en el desarrollo de los procesos sociales y cognitivos del alumnado.

Por otra parte, los centros docentes a través de la planificación de sus actuaciones educativas, tanto en los Planes de Formación, como por las experiencias de calidad de centro (O. EDU/1056/2014 de 4 de diciembre) muestran su interés por recibir formación en educación emocional o IE, Ilegando al 89\% de los cursos realizados.

\section{Conclusión}

El presente trabajo consciente de la importancia que la educación emocional tiene para el desarrollo integral de los alumnos de educación infantil ha tenido como objetivo analizar la oferta formativa que los CFIEs de Burgos y Miranda de Ebro ofrecen sobre esta temática al profesorado, como principal transmisor de estas competencias en el aula.

A lo largo de la investigación comprobamos como, en general, son muy pocos los programa socio-emocionales destinados a fomentar la IE del profesorado (Cabello et al., 2010; Fernández-Berrocal et al., 2008), si bien, nosotros encontramos un significativo incremento de la oferta formativa en competencias emocionales en los últimos años en los CFIEs analizados, especialmente desde el año 2014. Esto coincide con el interés y motivación creciente del profesorado, señalado ya por algunos autores, por dotar al alumnado no sólo de conocimientos sino también de habilidades sociales y emocionales (Brackett y Caruso, 2007; Cabello et al., 2010).

Otro de los aspectos que algunos autores destacan en la literatura es que este tipo de formación se produce de forma excesivamente teórica y poco vivencial y práctica (Cabello et al., 2010; Fernández et al., 2009; Palomero, 2009). En nuestro análisis observamos igualmente, que un importante porcentaje de la formación se lleva a cabo a través de seminarios o cursos, en lugar de grupos de trabajo, lo que confirma de alguna manera la falta de experiencia práctica que se señala en la literatura. Esto puede ser debido a la propia legislación educativa que regula esta formación. Por ejemplo, el artículo 8 de la Orden EDU/1057/2014, de 4 de diciembre, establece que la formación demandada se caracteriza por ser planificada por sujetos expertos en la materia y no por el propio grupo que solicita la formación, así como por no estipular como obligatorio un módulo de aplicación en el aula.

Por otro lado, se observa, como los profesores destinatarios de esta formación son de forma significativa maestros de Educación Infantil y/o Primaria, etapas clave en la adquisición de competencias emocionales si se analizan los contenidos trasversales especificados en los currículos de las etapas mencionadas. 
Por lo tanto, concluimos que la formación en competencias emocionales en los profesores de Educación Infantil es un aspecto clave que redunda en el desarrollo integral del alumnado. Sin embargo, pensamos al igual que otros autores (Gutiérrez et al., 2013) que no es posible enseñar una competencia que previamente no se ha alcanzado, al igual que no es posible enseñar con calidad ante la ausencia de bienestar docente (Ramírez y De la Herrán, 2012). Es decir, es difícil enseñar competencias emocionales, si previamente no ha existido una formación adecuada para ello. En este sentido, el interés por este tipo de formación va en aumento especialmente en los profesores de Educación Infantil y Primaria, potenciándose principalmente desde la escuela pública.

Por todo ello, es necesario que los responsables de la formación del profesorado se marquen como objetivo prioritario educar emocionalmente a los docentes, y en concreto, que planifiquen un plan de actuación a implementar en todas las zonas de la provincia de Burgos. Ya que como se ha señalado a lo largo de este trabajo, las repercusiones sobre los centros educativos y el alumnado son muy positivas (Conde y Almagro, 2013; Fernández-Berrocal y Extremera, 2002; Fernández-Berrocal et al., 2008; Sáenz-López Buñuel y Heras Pérez, 2013).

Las limitaciones del trabajo se centran en haber analizado en exclusiva la oferta formativa de los CFIEs de la provincia de Burgos, por lo que como investigaciones futuras sería interesante ampliar este estudio a nivel nacional. Igualmente, creemos importante analizar el contenido de los principales programas de educación emocional para docentes de Educación Infantil con el objetivo de detectar las potencialidades y las lagunas existentes en los mismos, así como el grado de satisfacción y el compromiso de aplicación en aula.

\section{RefERENCIAS BIBLIOGRÁFICAS}

Barberá, J. P. y Fuentes, M. (2012). Aprender inteligencia emocional mediante formación online ¿es posible?, 1-8. Recuperado de: http://www.raco.cat/index.php/ DIM/article/viewFile/269833/357359.

Barrantes, G., Casas, L. M. y Luengo, R. (2011). Obstáculos percibidos para la integración de las TIC por los profesores de Infantil y Primaria en Extremadura. PixelBit. Revista de Medios y Educación, 39, 83-94.

Brackett, M. A. y Caruso, D. R. (2006). The emotionally intelligent teacher. Ann Arbor, Michigan: QuestEducation.

Bisquerra, R. (2000). Educación emocional y bienestar. Barcelona: Praxis.

Bisquerra, R. (2003). Educación Emocional y Competencias Básicas para la vida. Revista de Investigación Educativa (RIE) 21(1), 7-43.

Bisquerra, R. (2005). La educación emocional en la formación del profesorado. Revista Interuniversitaria de Formación del Profesorado, 19(54), 95-114. Recuperado de: http://dialnet.unirioja.es/servlet/articulo?codigo=2126758.

Bisquerra, R. (2007). La educación emocional en la formación del profesorado. Revista Interuniversitaria de Formación del Profesorado, 54, 95-114. 
Cabello, R., Ruiz-Aranda, D. y Fernández-Berrocal, P. (2010). Docentes emocionalmente inteligentes. Revista Electrónica Interuniversitaria de Formación del Profesorado, 13(1), 41-49. Recuperado de: http://dialnet.unirioja.es/servlet/ articulo?codigo $=3163455$.

Cassullo, G. L. y García, L. (2015). Estudio de las Competencias Socio Emocionales y su Relación con el Afrontamiento en Futuros Profesores de Nivel Medio. Revista Electrónica Interuniversitaria de Formación del Profesorado, 18(1), 213-228. DOI: http://doi.org/10.6018/reifop.18.1.193041.

Conde, C. y Almagro, B. J. (2013). Estrategias para desarrollar la inteligencia emocional y la motivación en el alumnado de Educación Física. E-Motion: Revista de Educación, Motricidad e Investigación, 1, 212-220.

Damasio, A. R. (2001). El error de Descartes. Barcelona: Crítica.

De Andrés Viloria, C. (2005). La educación emocional en edades tempranas y el interés de su aplicación en la escuela. Programas de educación emocional, nuevo reto en la formación de los profesores. Tendencias Pedagógicas, 10, 5-17.

Decreto 51/2014, de 9 de octubre, por el que se regula la formación permanente del profesorado de enseñanzas no universitarias que presta sus servicios en centros docentes sostenidos con fondos públicos en la Comunidad de Castilla y León, BOCYL de 10 de octubre de 2014.

Durlak, J. A. y Weissberg, R. P. (2005). A major meta-analysis of positive youth development programs. Annual Meeting of the American Psychological Association, Washington, D. C., Estados Unidos, 18-21 agosto.

Extremera, N. y Femández-Berrocal, P. (2004a). La importancia de desarrollar la inteligencia emocional en el profesorado. Revista Iberoamericana de Educación, 33(8). Recuperado de: http://www.campus-ei.org/revista/deloslertores/759Extremera. PDF.

Extremera, N. y Fernández-Berrocal, P. (2004b). El papel de la inteligencia emocional en el alumnado: evidencias empíricas. Revista Electrónica de Investigación Educativa, 6(2). Recuperado de: http://redie.uabc.mx/vol6no2/contenido-extremera.html.

Extremera, N. y Fernández-Berrocal, P. (2004c). Inteligencia Emocional, calidad de las relaciones interpersonales y empatía en estudiantes universitarios. Clínica y Salud, 15(2), 117-137.

Extremera, N. y Fernández-Berrocal, P. (2005). Perceived emotional intelligence and life satisfaction: Predictive and incremental validity using the Trait Meta-Mood Scale. Personality and Individual Differences, 39(5), 937-948.

Fernández-Berrocal, P., Extremera, N. y Palomera, R. (2008). Emotional Intelligence as a crucial mental ability on educational context. En A. Valle y J. C. Nuñez (eds.), Handbook of Instructional Resources and their applications in the classroom. New York: Nova, 67-88.

Fernández-Berrocal, P. y Extremera, N. (2002). La inteligencia emocional como una habilidad esencial en la escuela. Revista Iberoamericana de Educación, 29, 1-6.

Fernández, M. R., Palomero, J. E. y Teruel, M. P. (2009). El desarrollo socioafectivo en la formación inicial de los maestros. Revista Interuniversitaria de Formación del Profesorado, 12(1), 33-50. 
García Martínez, J., Fernández Ozcorta, E. J., Rodríguez Peláez, D. y Tornero Quiñones, I. (2013). Necesidades formativas en competencias socioemocional en el cuerpo docente. E-Motion: Revista de Educación, Motricidad e Investigación, 1, 128143. Recuperado de: http://dialnet.unirioja.es/servlet/articulo?codigo $=4707706$.

Goleman, D. (1995). Emotional intelligence: why it can matter more tan IQ? Nueva York: Bantam Books.

Gutiérrez, M., Ibáñez, R. y Aguilar Moya, R. (2013). Apuesta por la formación de competencias desde la inteligencia emocional: reflexiones sobre su importancia en la docencia. Edetania: Estudios y Propuestas Socio-Educativas, 44, 77-92. Recuperado de: http://dialnet.unirioja.es/servlet/articulo?codigo=4596149.

Jennings, P. A. y Greenberg, M. T. (2009). The Prosocial Classroom: Teacher Social and Emotional Competence in Relation to Student and Classroom Outcomes. Review of Educational Research, 79(1), 491-525.

López-Goñi, I. y Goñi, J. M. (2012). La competencia emocional en los currículos de formación inicial de los docentes. Un estudio comparativo. Revista de Educación, 357, 205-206.

Mayer, J. D. y Salovey, P. (1997). What is emotional intelligence? En P. Salovey y D. Sluyter (eds.), Emotional development and emotional intelligence: implications for educators. Nueva York: Basic Books, 3-31.

Mayer, J. D., Salovey, P. y Caruso, D. (2000). Models of emotional intelligence. En R. J. Sternberg (eds.). Handbook of intelligence (pp. 396-420). Nueva York: Cambridge University Press.

Molero, D., Ortega, F. y Moreno, M. R. (2010). Diferencias en la adquisición de competencias emocionales en función del género. Revista Electrónica de Investigación y Docencia, 3, 165-172.

Montero, I. y León, O. G. (2007). A guide for naming research studies in Psychology. International Journal of Clinical and HealthPsychology, 7(3), 847-862.

ORDEN EDU/1056/2014, de 4 de diciembre, por la que se regula la organización y funcionamiento de la Red de formación y la planificación, desarrollo y evaluación de la formación permanente del profesorado de enseñanzas no universitarias que presta sus servicios en centros docentes sostenidos con fondos públicos en la Comunidad de Castilla y León, BOCYL de 16 de diciembre de 2014.

ORDEN EDU/1057/2014, de 4 de diciembre, por la que se regulan las modalidades, convocatoria, reconocimiento, certificación y registro de las actividades de formación permanente del profesorado de enseñanzas no universitarias que presta sus servicios en centros docentes sostenidos con fondos públicos en la Comunidad de Castilla y León organizadas por la Red de formación y se establecen las condiciones de reconocimiento de las actividades de formación organizadas por otras entidades, BOCYL de 16 de diciembre de 2014.

Palomera, R., Fernández-Berrocal, P. y Brackett, M. A. (2008). La inteligencia emocional como una competencia básica en la formación inicial de los docentes: algunas evidencias. Revista Electrónica de Investigación Psicoeducativa, 15(6), 437-454.

Palomero Pescador, J. E. (2005). La Educación Emocional, una revolución pendiente. Revista Interuniversitaria de Formación del Profesorado, 19(3), 9-13. Recuperado de: http://dialnet.unirioja.es/servlet/articulo?codigo=2126747. 
Palomero, P. (2009). Desarrollo de la competencia social y emocional del profesorado: una aproximación desde la psicología humanista. Revista Interuniversitaria de Formación del Profesorado, 12(2), 145-153. Recuperado de: http://dialnet. unirioja.es/servlet/articulo?codigo $=2126747$.

Pegalajar Palomino, M. C. y López Hernáez, L. (2015). Competencias Emocionales en el Proceso de Formación del Docente de Educación Infantil Emotional Competences in the Childhood Education Teacher Training. REICE. Revista Iberoamericana sobre Calidad, Eficacia y Cambio en Educación, 13(3), 95-106.

Poulou, M. (2005). The Prevention of Emotional and Behavioural Difficulties in Schools: Teachers' suggestions. Educational Psychology in Pratice, 21, 37-52.

Punset, E. (2010). Viaje a las emociones. Las claves que mueven el mundo: la felicidad, el amor y el poder de la mente. Barcelona: Destino.

Qualter, P., Gardner, K. J., Pope, D. J. y Kelly, J. M. (2012). Ability emotional intelligence, trait emotional intelligence, and academic success in British secondary schools: A 5 year longitudinal study. Learning and Individual Differences, 22(1), 83-91.

Ramírez, M. S. y De la Herrán, A. (2012). La madurez personal en el desarrollo profesional del docente. REICE. Revista Iberoamericana sobre Calidad, Eficacia y Cambio en Educación, 10(3), 25-44.

Sáenz-López Buñuel, P., y Heras Pérez, M. A. (2013). ¿Por qué y cómo plantear la educación emocional como el reto del siglo XXI? E-Motion: Revista de Educación, Motricidad e Investigación, 1, 67-82.

Shapiro, L. E. (1998). La inteligencia emocional de los niños. Barcelona: Ediciones B. 